\title{
Sonographic appearance of Youssef's syndrome: A case report and literature review
}

\author{
Etedafe P. Gharoro ${ }^{1 *}$, Ehigha J. Enabudoso ${ }^{1}$, Edafe E. Gharoro ${ }^{2}$ \\ ${ }^{1}$ Department of Obstetrics and Gynaecology, University of Benin Teaching Hospital, Benin City, Nigeria \\ ${ }^{2}$ PEPFAR, University of Benin Teaching Hospital, Benin City, Nigeria \\ Email: "gharoro@uniben.edu, ${ }^{*}$ gharoro@hotmail.com
}

Received 28 June 2013; revised 30 July 2013; accepted 7 August 2013

Copyright (C) 2013 Etedafe P. Gharoro et al. This is an open access article distributed under the Creative Commons Attribution License, which permits unrestricted use, distribution, and reproduction in any medium, provided the original work is properly cited.

\begin{abstract}
Vesico-vaginal fistula is still a major problem in the developing world, in particular Nigeria. Vesico-uterine fistula (Youssef's syndrome) following relief of obstructed labor by caesarean section is commoner than previously thought. We report a case of Mrs U.B. a 28-year-old $\mathrm{P} 2+^{0}$ with one living child, who presented with a history of $2^{0}$ amenorrhea and infertility of 8 years duration. She had caesarean section to relieve an obstructed labor with macerated stillbirth in her last pregnancy. As part of her routine investigation for $2^{0}$ amenorrhea and infertility, a request for ultrasound scan was made. Ultrasound pictures are presented and discussed. Standard diagnostic investigations for Youssef's syndrome are by cystoscopy, intravenous urogram and or hysterosalpingography, sonodiagnosis (sonohysterography) is primarily not resorted. We recommend as a standard investigation tool, Ultrasound scan (Sonodiagnosis) for all patients with Vesicovaginal fistula.
\end{abstract}

Keywords: Vesico-Vaginal Fistula; Vesico-Uterine Fistula; Youssef's Syndrome; Ultrasound Scan;

Sonohysterography

\section{INTRODUCTION}

\subsection{Background}

Vesico-vaginal fistula is still a major problem in the developing world, in particular Nigeria. Vesicouterine fistula (Youssef's syndrome) following relief of obstructed labor by caesarean section is commoner than previously thought. Standard diagnostic investigations for vesicouterine fistula are by cystoscopy, intravenous urogram and or hysterosalpingography, sonodiagnosis (sonohysterography) is primarily not resorted.

${ }^{*}$ Corresponding author.

\subsection{Case Report}

We report a case of Mrs U.B. a 28-year-old P2 $+{ }^{0}$ with one living child, who presented with the history of $2^{0}$ amenorrhea and infertility of 8 years duration. In the year 2004 during her last pregnancy and delivery, she had caesarean section to relieve an obstructed labor with macerated stillbirth. Post operative period was complicated by wound sepsis and breakdown.

She has visited numerous health facilities for treatment of her amenorrhea with no successful outcome. On presentation she had no urinary incontinence and has not observed any haematuria. As part of her routine investigation for $2^{0}$ amenorrhea and infertility, a request for ultrasound scan was made. A scan was done using a 3D ultrasound scan machine (Samsung MySonoU5 with a 3D2-6 probe).

Figure 1 shows the urinary bladder partially filled with urine with a fistulous communication between the uterus and (L) adnexia (Arrowed-white). The uterine endometrial cavity is visualized distended with urine (hypoechoic collection). The cervix is completely closed by fibrosis seen as hyperechoic structure.

Figure 2 is a long axis scan of the pelvis, which shows an anteverted uterus filled with clear fluid, measuring $8.86 \mathrm{~cm} \times 3.86 \mathrm{~cm}$ with a fistula (Arrowed-white) between the anterior lower uterine segment wall and the bladder. There is moderate amount of fluid present in the Pouch of Douglas.

\section{DISCUSSION}

Worldwide Vesico-uterine fistula (VUF) is a rare complication of caesarean section $[1,2]$. In our practice environment caesarean section contribution to obstetric fistula incidence is high [3-5]. Mrs. U.B. did not present with the three classical triad of Youssef's syndrome: urinary continence, cyclical haematuria (menouria), and amenorrhea [6,7]. Vesico-uterine fistula usually present 
in three classical forms. She presented with features of type 1 Vesico-uterine fistula (VUF) [7]. The other two types have variable amount of urinary incontinence due to a patent lower uterine segment and cervical canal.

The clinical problem of Vesico-uterine fistula in this patient was unrecognized as urinary incontinence was not her problem and a diagnosis was not made for 8 years of her seeking medical help. Patients' standard investigations for Vesico-vaginal fistula (VVF) usually include cystoscopy, intravenous urogram and or hysterosalpingography [8], ultrasound scan as an investigative tool is primarily not resorted.

A high-resolution ultrasound scan (volume scan) clearly demonstrated the abnormal communications between the bladder, uterus and parametrium. The ultrasound scan shortened the diagnostic investigation of the patient. In addition the cost of investigation and

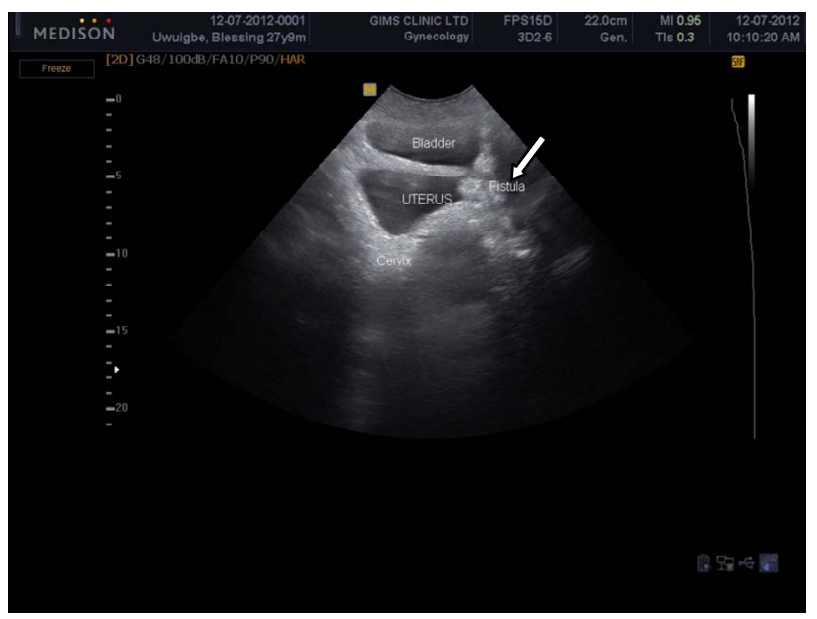

Figure 1. Bladder partially filled with urine with a fistula communication between the uterus and (L) adnexia. The uterine endometrial cavity is distended with urine. The cervix is tightly closed and fibrosed.

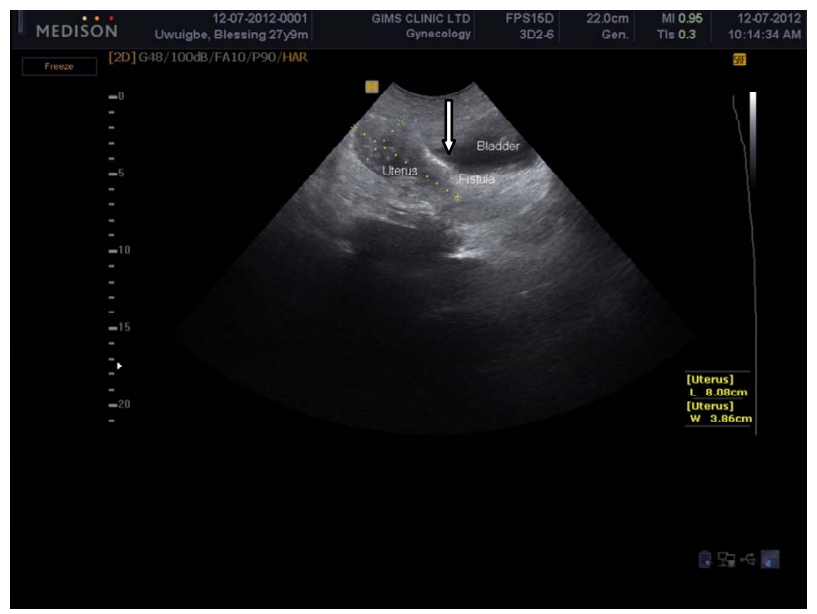

Figure 2. Anteverted uterus filled with clear fluid with a fistula communication between the cervical canal and the bladder. exposure to irradiation from either hysterosalpingography or intravenous urogram was eliminated. Ultrasound scan is cheaper and safer comparatively. Other workers have demonstrated the use of ultrasound scan (sonohysterography) as an easy and feasible diagnostic method for investigating vesicouterine fistula $[9,10]$.

\section{CONCLUSION}

Pelvic, renal and general abdominal scan (Sonodiagnosis) is a suitable diagnostic tool for the routine assessment of all patients with VVF. Ultrasound scanning at point of care for patient with complex gynecological morbidities including Vesico-vaginal fistula by practicing obstetricians and gynecologists is cost effective, beneficial and time saving to patients.

\section{CONFLICT OF INTEREST NOTIFICATION}

The authors wish to state that there is no conflict of interest in execution and publishing of this project. The authors privately funded the research.

\section{REFERENCES}

[1] Youssef, A.F. (1957) Menouria following lower segment cesarean section; a syndrome. American Journal of $\mathrm{Ob}$ stetrics \& Gynecology, 73, 759-767.

[2] Rao, M.P., Dwivedi, U.S., Datta, B., Vyas, N., Nandy, P.R., Trivedi, S. and Singh, P.B. (2006) Post caesarean vesicouterine fistulae-Youssef's syndrome: Our experience and review of published work. ANZ Journal of Surgery, 76, 243-245. doi:10.1111/j.1445-2197.2006.03591.x

[3] Gharoro, E.P. and Abedi, H.O. (1999) Vesico-vaginal fistula in Benin City, Nigeria. International Journal of Gynecology \& Obstetrics, 64, 313-314. doi:10.1016/S0020-7292(98)00134-9

[4] Iloabachie, G.C. and Njoku, O. (1985) Vesico-uterine fistula. British Journal of Urology, 57, 438-439. doi:10.1111/j.1464-410X.1985.tb06305.x

[5] Ikechebelu, J.I., Ugboaja, J.O. and Okeke, C.F. (2011) Post-cesarean vesicouterine fistula (Youssef's syndrome): Report of two cases. Journal of Obstetrics and Gynaecology Research, 37, 912-915. doi:10.1111/j.1447-0756.2010.01428.x

[6] Dubuisson, J.B., Barbot, J. and Santarelli, J. (1979) Vesico-uterine fistulae after Caesarean operation. Journal de Gynecologie, Obstetrique et Biologie de la Reproduction (Paris), 8, 229-233.

[7] Józwik, M. and Józwik, M. (2000) Clinical classification of vesicouterine fistula. International Journal of Gynecology \& Obstetrics, 70, 353-357. doi:10.1016/S0020-7292(00)00247-2

[8] el Fassi, M.J., Tazi, K., Karmouni, T., Koutani, A., Ibn Attya, A.A., Hachimi, M. and Lakrissa, A. (2003) Youssef's syndrome (vesico-uterine fistula): Three case re- 
ports. Annales d Urologie (Paris), 37, 184-186. doi:10.1016/S0003-4401(03)00052-4

[9] Fenkci, I.V., Demirbas, M. and Oztekin, O. (2010) Sonohysterography in evaluation of Youssef's syndrome. International Urogynecology Journal, 21, 607-608. doi:10.1007/s00192-009-1029-0

[10] Bieńkiewicz, A. and Suzin, J. (1992) Ultrasound diagnosis of vesico-uterine fistula. Ginekologia Polska, 63, 534538 . 